\title{
THE UNUSUAL HYDROCARBON EMISSION FROM THE EARLY CARBON STAR HD 100764: THE CONNECTION BETWEEN AROMATICS AND ALIPHATICS
}

\author{
G. C. Sloan, ${ }^{1}$ M. Jura, ${ }^{2}$ W. W. Duley ${ }^{3}$ K. E. Kraemer,${ }^{4}$ J. Bernard-Salas, ${ }^{1}$ W. J. Forrest, ${ }^{5}$ \\ B. Sargent, ${ }^{5}$ A. Li, ${ }^{6}$ D. J. Barry, ${ }^{1}$ C. J. Bohac, ${ }^{5}$ D. M. Watson, ${ }^{5}$ and J. R. HoucK ${ }^{1}$ \\ Received 2006 December 23; accepted 2007 April 9
}

\begin{abstract}
We have used the Infrared Spectrograph (IRS) on the Spitzer Space Telescope to obtain spectra of HD 100764, an apparently single carbon star with a circumstellar disk. The spectrum shows emission features from polycyclic aromatic hydrocarbons (PAHs) that are shifted to longer wavelengths than normally seen, a characteristic of "class C" systems in the classification scheme of Peeters et al. All seven of the known class C PAH sources are illuminated by radiation fields that are cooler than those which typically excite PAH emission features. The observed wavelength shifts are consistent with hydrocarbon mixtures containing both aromatic and aliphatic bonds. We propose that the class C PAH spectra are distinctive because the carbonaceous material has not been subjected to a strong ultraviolet radiation field, allowing relatively fragile aliphatic materials to survive.
\end{abstract}

Subject headings: circumstellar matter — stars: carbon

Online material: color figures

\section{INTRODUCTION}

Complex hydrocarbons are found in a wide variety of astrophysical environments. Gillett et al. (1973) first detected what became known as the unidentified infrared (UIR) emission features, the strongest of which are at 3.3, 6.2, 7.7-7.9, 8.6, 11.3, and $12.7 \mu \mathrm{m}$. Leger \& Puget (1984) and Allamandola et al. (1985) identified the carrier of these features as polycyclic aromatic hydrocarbons (PAHs). The debate over PAHs as the carrier of the UIR features raged for many years, and while the identification now seems firm (e.g., Allamandola et al. 1999), many questions still remain over the origin, evolution, and exact composition of astrophysical hydrocarbons.

Peeters et al. (2002) used data from the Short Wavelength Spectrometer (SWS) on the Infrared Space Observatory (ISO) to group PAH spectra into three classes based on the positions of peak emission and profiles of the $\mathrm{C}-\mathrm{C}$ skeletal deformation modes within the PAHs at 6.2 and 7.7-7.9 $\mu \mathrm{m}$. The "class A" sources display features at 6.22 and $7.65 \mu \mathrm{m}$ and generally include $\mathrm{H}$ II regions, reflection nebulae, galaxies, and Herbig $\mathrm{Ae} / \mathrm{Be}(\mathrm{HAeBe})$ stars still embedded in the regions where they formed. The "class B" sources, which typically include planetary nebulae and isolated HAeBe stars, show these same features, but shifted to 6.26 and $7.85 \mu \mathrm{m}$. Peeters et al. (2002) also identified two "class C" sources, including the well-known Egg Nebula (AFGL 2688), which are in transition from the asymp-

\footnotetext{
1 Cornell University, Astronomy Department, Ithaca, NY 14853-6801; sloan@ isc.astro.cornell.edu,jbs@isc.astro.cornell.edu,don@isc.astro.cornell.edu,jrh13@ cornell.edu.

2 Department of Physics and Astronomy and Center for Astrobiology, University of California, Los Angeles, CA 90095-1562; jura@astro.ucla.edu.

3 Department of Physics and Astronomy, University of Waterloo, 200 University Avenue West, Waterloo, ON N2L 3G1, Canada; wwduley@uwaterloo.ca.

4 Air Force Research Laboratory, Space Vehicles Directorate, 29 Randolph Road, Hanscom AFB, MA 01731; kathleen.kraemer@hanscom.af.mil.

5 Department of Physics and Astronomy, University of Rochester, Rochester, NY 14627-0171; forrest@pas.rochester.edu, bsargent@pas.rochester.edu, cbohac@ pas.rochester.edu,dmw@pas.rochester.edu.

6 Department of Physics and Astronomy, University of Missouri, Columbia, MO65211; lia@missouri.edu.
}

totic giant branch (AGB) to the planetary nebula phase. These two sources show emission at $6.26 \mu \mathrm{m}$, no emission near $7.65 \mu \mathrm{m}$, and a broad feature centered at $\sim 8.2 \mu \mathrm{m}$. It remains unclear how these different classes of PAHs arise in different astrophysical environments.

Observations with the Infrared Spectrograph (IRS; Houck et al. 2004) on the Spitzer Space Telescope (Werner et al. 2004) have added to the small sample of class C PAH spectra. Kraemer et al. (2006) reported the detection of a class C PAH spectrum from MSX SMC 029, a transition object in the Small Magellanic Cloud (SMC). Jura et al. (2006) detected a class C PAH spectrum from HD 233517, an apparently single red giant (K2 III) with a substantial infrared excess, most likely arising from a circumstellar disk (Jura 2003). This system is remarkable because some of the hydrocarbons were probably synthesized from the oxygen-rich disk.

HD 100764 is one of a handful of red giants (luminosity class III) with large infrared excesses, characteristic of orbiting disks. While most of these stars are oxygen-rich like HD 233517 , HD 100764 is carbon-rich. As part of a program to better understand these stars, we obtained IRS data for HD 100764. We have found that its IRS spectrum reveals low-contrast class C PAH features not detectable in previous ground-based spectroscopy. In this paper, we describe the PAH spectrum of HD 100764 in detail and relate it to other class $\mathrm{C}$ PAH sources.

HD 100764, also known as CCS 1886 and CGCS 3066, is classified as an early carbon star, R2 (Sanford 1944) or C1,1 (Yamashita 1972). Using optical spectroscopy, Dominy (1984) found that the central star has a temperature of $4850 \mathrm{~K}$, is iron poor, and has an enhanced ${ }^{13} \mathrm{C} /{ }^{12} \mathrm{C}$ ratio, making this carbon star one of the uncommon J stars. The Hipparcos parallax of $2.78 \pm$ 1.18 mas yields an uncertain distance of $\sim 360 \mathrm{pc}$ and thus a total luminosity of $73 L_{\odot}$ (Parthasarathy 1991), indicating that the star is a first-ascent red giant.

Lloyd Evans (1990) identified HD 100764 as a potential silicate/carbon star because of its red [12] - [25] color, as measured by the Infrared Astronomy Satellite (IRAS). As a consequence, it was observed twice using ground-based mid-infrared spectrometers over a decade ago. LeVan et al. (1992) obtained an 8-14 $\mu \mathrm{m}$ 
TABLE 1

Low-Resolution Wavelength Ranges

\begin{tabular}{|c|c|c|}
\hline Order & $\begin{array}{c}\mathrm{SL} \\
(\mu \mathrm{m})\end{array}$ & $\begin{array}{c}\mathrm{LL} \\
(\mu \mathrm{m})\end{array}$ \\
\hline Second.. & $5.10-7.59$ & $14.20-21.23$ \\
\hline ….................. & $7.73-8.39$ & $19.28-21.23$ \\
\hline …................ & $7.59-14.33$ & $20.46-37.00$ \\
\hline
\end{tabular}

spectrum at the Wyoming Infrared Observatory (WIRO) with a resolution $(\lambda / \Delta \lambda)$ of $\sim 50$ and a signal/noise $(\mathrm{S} / \mathrm{N})$ ratio of $\sim 5$. While they could rule out the presence of silicate dust emission at $10 \mu \mathrm{m}$ in the spectrum, they could say little more. Skinner (1994), who obtained a higher quality spectrum with CGS3 at the United Kingdom Infrared Telescope (UKIRT), also saw no spectral features from dust and concluded that the infrared emission was dominated by amorphous carbon dust in a circumstellar disk. The disk explains why the strong infrared excess does not appreciably redden the optical spectrum.

Although uncertain, it is plausible that this disk formed when HD 100764 became a red giant and engulfed a companion. The luminosity of HD 100764 is consistent with other R stars with measured parallaxes, which Alksnis et al. (1998) have determined are first-ascent red giants, not AGB stars. McClure (1997) observed that $\mathrm{R}$ stars do not have companions and suggested that their carbon-rich nature and the lack of a companion could both be explained by coalescence of an expanding giant and its companion.

\section{THE INFRARED SPECTRA}

\subsection{Observations and Analysis}

We observed HD 100764 with both the Short-Low (SL) and Long-Low (LL) modules of the IRS in standard staring mode on 2006 January 14. Because the source is bright for the IRS, we used the shortest possible ramps in both modules, $6 \mathrm{~s}$ ramps which provide only four samples. This source was observed independently with the Short-High (SH) and Long-High (LH) modules in Spitzer program 278 (AOR key 19481344), and we include those data here with permission of the PI, M. Werner.

We followed the standard calibration method used at Cornell. We started with the S14 pipeline output from the Spitzer Science Center (SSC) and subtracted a sky image from each image before extracting a spectrum. The high-resolution observations include separate sky images obtained close to the target. For SL, the sky image for one spectral aperture is obtained when the source is in the other aperture (i.e., aperture differences SL2-SL1 and SL1SL2, where "SL2" means "SL Order 2"). For LL, we simply differenced the images with the source in the same aperture, but in the other nod position. For a source as bright as HD 100764 , the primary benefit of background removal is the subtraction of most of the rogue pixels. These pixels have temporarily increased dark currents and are the primary source of noise in images with bright sources. We applied imclean. pro $^{7}$ to replace remaining rogue and bad pixels.

Spectra were extracted from the individual images using the SSC-defined apertures and the routines profile, ridge, and extract, which are available with the SPICE software package (Spitzer/IRS Custom Extraction). To calibrate to flux-density units, we used a spectral correction generated from IRS obser-

\footnotetext{
${ }^{7}$ Available from the SSC as irsclean. pro.
}

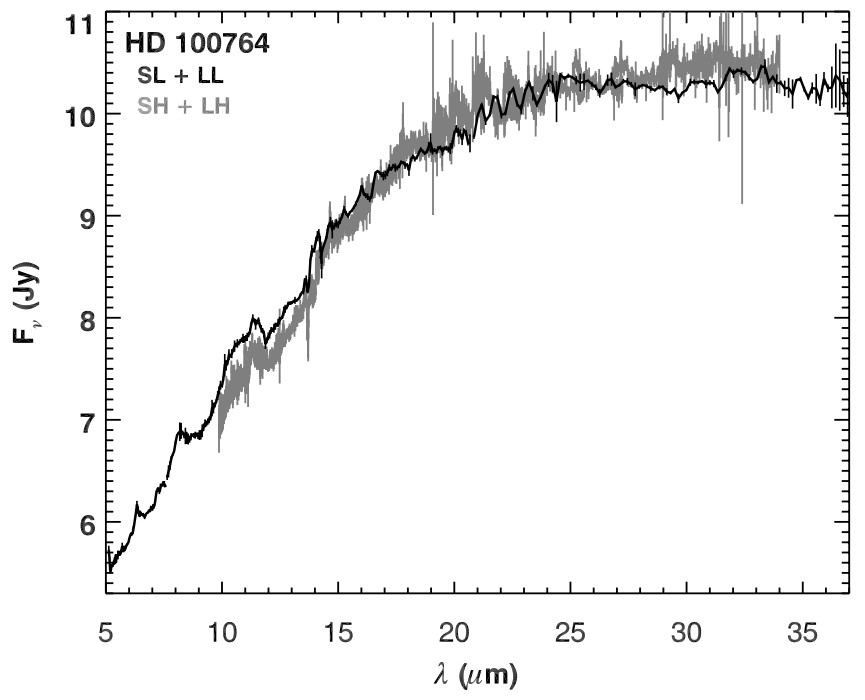

FIG. 1.-Spectrum of HD 100764 with the low-resolution modules (black) and with the high-resolution modules (gray). [See the electronic edition of the Journal for a color version of this figure.]

vations of standard stars: HR 6348 (K0 III) for SL, and HR 6348, HD 166780 (K4 III), and HD 173511 (K5 III) for LL. The highresolution data were calibrated using $\xi$ Dra (K2 III). Error bars are based on the standard deviation of the spectrum in the two separate nods in each aperture. The resulting spectrum has a $\mathrm{S} / \mathrm{N}$ ratio typically between 200 and 400 .

We applied scalar multiplicative corrections to the segments to remove discontinuities and align them upwards to the presumably best-centered spectral segment, in this case LL2. This step required corrections of $9 \%$ to SL2, $2 \%$ to SL1, and $1 \%$ to LL1. These corrections indicate the accuracy of the pointing of the telescope and are consistent with typical observations. Finally, we combined the bonus order with valid data in the overlapping orders and removed all data outside the ranges of trustworthy signal. Table 1 gives the wavelength ranges used for each lowresolution order.

The flux density from HD 100764 in the 5-37 $\mu \mathrm{m}$ spectral region ranges between 5 and $11 \mathrm{Jy}$, and we have therefore assessed the saturation and nonlinearities in the response function, especially in LL. The S14 pipeline has a greatly improved correction for these problems, and it produces much better spectra compared to previous pipeline versions. Figure 1 presents the full spectrum of HD 100764, both our low-resolution data and also the high-resolution data obtained by $\mathrm{M}$. Werner et al. A comparison of the two spectra reveals no evidence for any residual artifacts from the saturation and linearity corrections in our lowresolution spectrum. One artifact that does remain is the fringing from the LL1 filter apparent between 21 and $25 \mu \mathrm{m}$. We do not attempt a correction, because the analysis in this paper concentrates on the features at shorter wavelengths. For this paper, we focus on the low-resolution spectrum from 5 to $14 \mu \mathrm{m}$.

Superimposed on the continuum produced by the circumstellar disk are emission features at wavelengths near those associated with PAHs and a broad emission feature centered at $10.6 \mu \mathrm{m}$. To isolate the PAH-related features, we subtracted a spline-fitted continuum, as Figure 2 shows. This spline passes through and includes the broad feature peaking at $10.63 \mu \mathrm{m}$. We examine this feature separately in $\S 2.5$. The spectrum of HD 100764 also shows a narrow absorption band from acetylene $\left(\mathrm{C}_{2} \mathrm{H}_{2}\right)$ at $13.7 \mu \mathrm{m}$. When this absorption band appears with $\mathrm{PAH}$ emission features, it is only in the rare class C PAH spectra (Kraemer et al. 2006). 


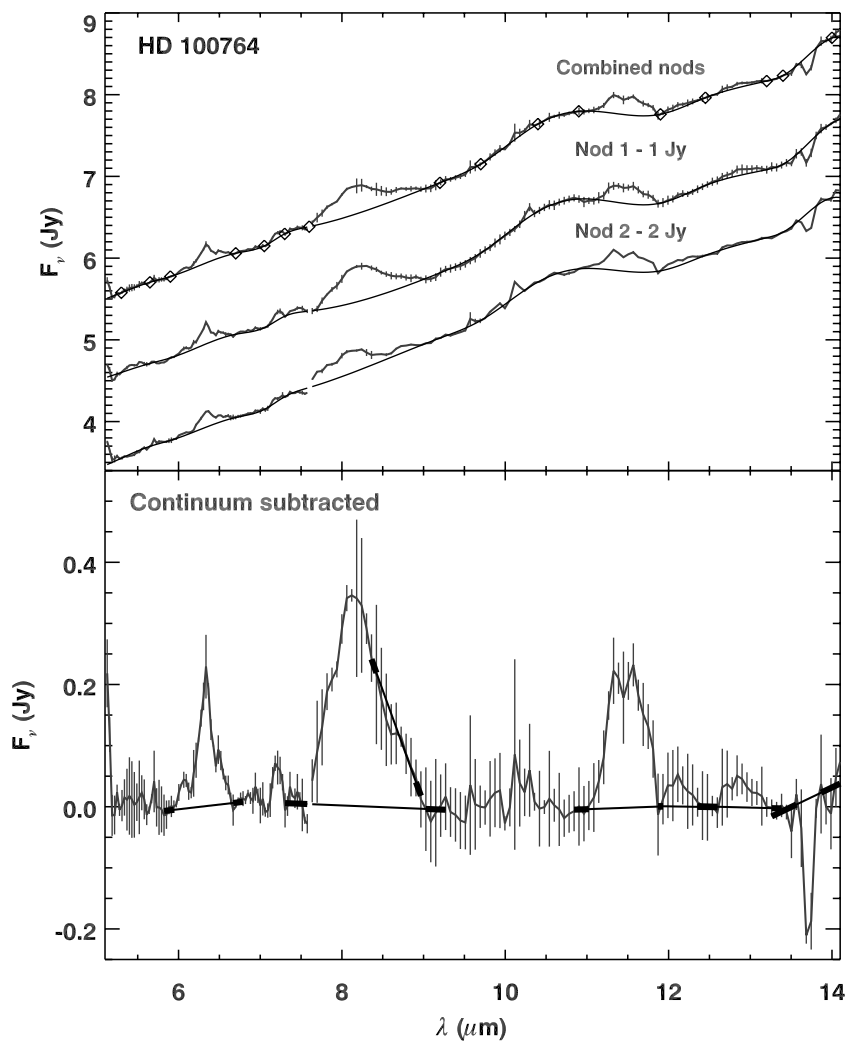

FIG. 2.- Spectrum of HD 100764. The top panel shows the co-added spectrum and the spectra from the individual nod positions (offset by 1 and $2 \mathrm{Jy}$, respectively). The thin solid curve is a spline fit to the data at wavelengths marked with diamonds. These points lie outside of the PAH features and $\mathrm{C}_{2} \mathrm{H}_{2}$ absorption band at $13.7 \mu \mathrm{m}$. The bottom panel shows the resulting continuum-subtracted spectrum, along with the error bars and the line segments fit to extract the strengths and centers of the spectroscopic features. The line segments are thick in the wavelength regions used to fit the continuum and thin where the feature is integrated. The equivalent width of the $13.7 \mu \mathrm{m}$ acetylene band is measured before any continuum subtraction. [See the electronic edition of the Journal for a color version of this figure.]

The low-contrast features in the $8-14 \mu \mathrm{m}$ range escaped previous detection. The $8 \mu \mathrm{m}$ feature has a contrast of $5 \%$, but lies at the edge of the ground-based window, and the feature near $11.3 \mu \mathrm{m}$ has a contrast of only $3 \%$, too small to be seen even in the spectrum by Skinner (1994), which has a S/N ratio of $\sim 15$ at those wavelengths.

Figure 2 (top) plots the spectra from the two nod positions separately. Systematic errors in the spectra have their greatest impact on low-contrast features, and they often show up as dif- ferences between the nods. Some detailed differences in the PAH features are apparent, most notably at $\sim 8.5 \mu \mathrm{m}$, but these differences are not large enough to have a significant quantitative effect on our conclusions. Figure 2 (bottom) presents the continuum-subtracted spectrum from HD 100764, along with line segments used to extract the strength and position of each PAH emission feature, as described in $\S 2.3$.

\subsection{The Comparison Sample}

To place the class C PAH spectrum from HD 100764 in context, we have also examined several other PAH spectra. These comparison spectra include the two class C PAH sources from the SWS database, AFGL 2688 and IRAS 13416-6243, along with SWS spectra of NGC 1333 SVS 3 and HD 44179 (the Red Rectangle). These sources are prototypical examples of class A and B PAH spectra, respectively. All four spectra are from the atlas of SWS spectra produced by Sloan et al. (2003), and they have been regridded to a lower spectral resolution. In the case of AFGL 2688, the spectrum is an average of two observations. The comparison sample also includes the four sources observed by the IRS which are currently known to have PAH spectra either in or near class C, including the K giant HD 233517 (Jura et al. 2006), the post-AGB source MSX SMC 029 (Kraemer et al. 2006), the HAeBe source HD 135344 (Sloan et al. 2005), and the intermediate-mass T Tauri star SU Aur (Furlan et al. 2006). Table 2 presents the comparison sample, including their spectral classes and effective temperatures, which are described in $\S 3.1$.

\subsection{Extracting the PAH Features}

For each source, we follow the algorithm described for HD 100764 by spline fitting and removing a continuum, as Figure 3 illustrates. It has proven necessary to shift the spline points from one spectrum to the next to avoid some of the spectral structure which only appears in individual spectra. Care must be taken in the cases of IRAS 13416 and AFGL 2688, because the PAH features have such low contrast with respect to the continuum. In the case of AFGL 2688, the features at 12.0 and $12.7 \mu \mathrm{m}$ must be treated with caution, because their apparent strength and position shift substantially with the choice of spline points. The spline fit to SU Aur removes the silicate emission feature; this method has been tested for several similar sources showing both PAH and silicate emission by Keller et al. (2007). Two of the spectra have been smoothed: MSX SMC 029 with a 3 pixel boxcar and HD 233517 with a 5 pixel boxcar. Figure 4 presents the continuum-subtracted PAH spectra for HD 100764 and the comparison sample.

TABLE 2

HD 100764 AND the Comparison SAmple

\begin{tabular}{|c|c|c|c|c|c|c|}
\hline Target & Instrument & AOR Key or TDT & Object Type $^{\mathrm{a}}$ & Spectral Type ${ }^{a}$ & $\begin{array}{l}T_{\text {eff }}{ }^{a} \\
(\mathrm{~K})\end{array}$ & PAH Class \\
\hline 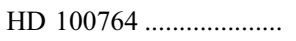 & IRS & 16262656 & Red giant & $\mathrm{C} 1,1$ & 4850 & $\mathrm{C}$ \\
\hline IRAS $13416-6243 \ldots \ldots \ldots$ & SWS & 62803904 & Post-AGB & G1 I & 5440 & $\mathrm{C}$ \\
\hline 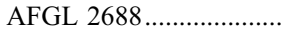 & SWS & 33800604,35102563 & Post-AGB & F5 Ia & 6520 & $\mathrm{C}$ \\
\hline MSX SMC $029 \ldots \ldots \ldots \ldots \ldots . . . .$. & IRS & 10656256 & Post-AGB & & & $\mathrm{C}$ \\
\hline HD $233517 \ldots \ldots \ldots \ldots \ldots . .$. & IRS & 3586048 & Red giant & K2 III & 4475 & $\mathrm{C}$ \\
\hline 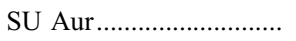 & IRS & 3533824 & $\mathrm{~T}$ Tauri & G1 & 5945 & $\mathrm{C}$ \\
\hline 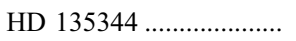 & IRS & 3580672 & Herbig Ae/Be & $\mathrm{F} 4 \mathrm{Ve}$ & 6590 & $\mathrm{~B} / \mathrm{C}$ \\
\hline 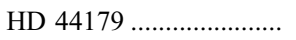 & SWS & 70201801 & Post-AGB & A0 III & 9520 & $\mathrm{~B}$ \\
\hline NGC 1333 SVS $3 . . . \ldots \ldots . .$. & SWS & 65902719 & Reflection nebula & B6 & 14,000 & A \\
\hline
\end{tabular}

${ }^{\text {a }}$ See $\S 3.1$ for details and references. 


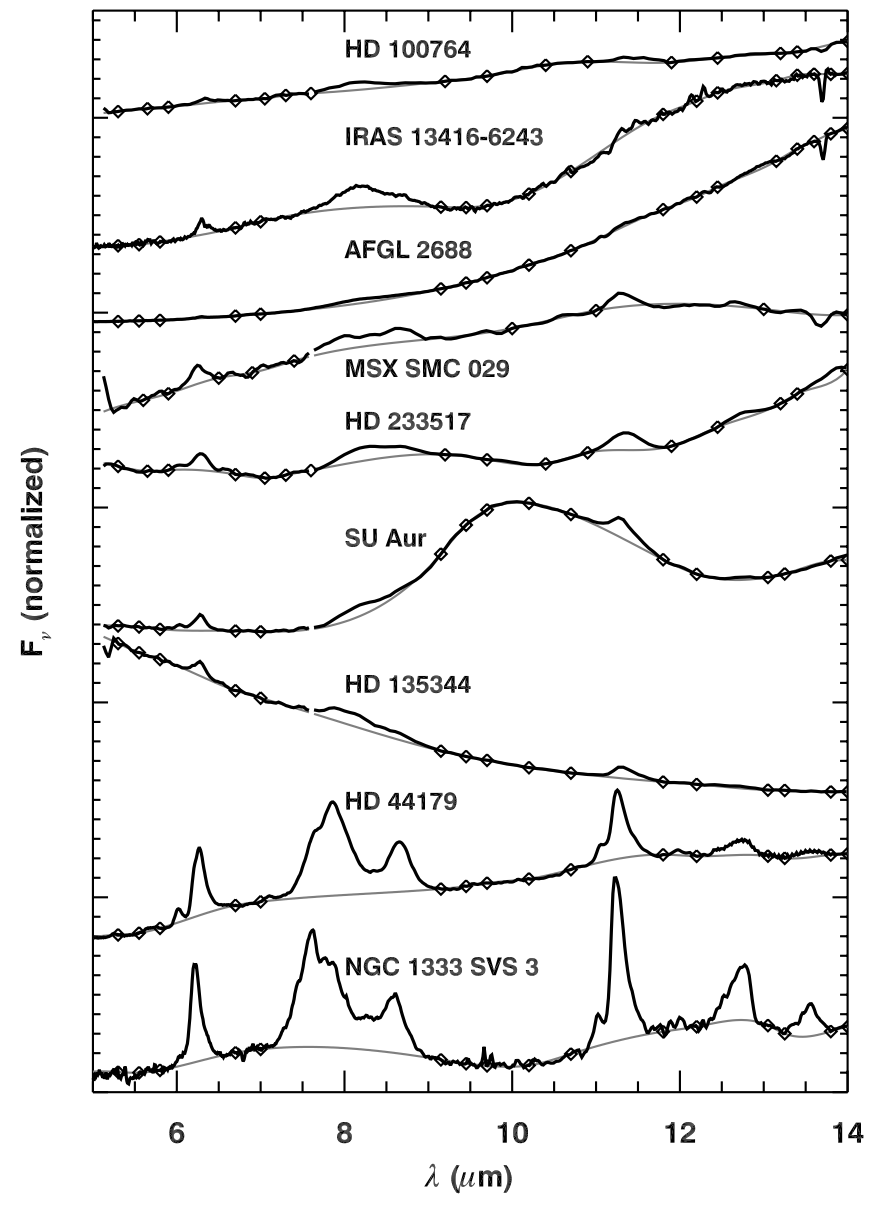

Fig. 3.- Infrared spectra of several sources showing class C PAH features, along with spectra of HD 44179 (class B) and NGC 1333 SVS 3 (class A). The spectra of IRAS 13416, AFGL 2688, NGC 1333 SVS 3, and HD 44179 are from the $I S O /$ SWS database (as processed by Sloan et al. 2003); the remaining spectra are from the IRS. The thin lines show the continua fit to the spectra, based on a spline through the wavelengths marked with diamonds. The resulting continuumsubtracted PAH spectra appear in Fig. 4. [See the electronic edition of the Journal for a color version of this figure.]

Fitting and removing a spline removes most, but not all, of the continuum spectrum. Because we have not forced the fitted spline to pass precisely through the continuum on either side of each feature, small residual tilts remain. While these would have little impact on the extracted strengths of the features, they would shift the measured center. We therefore continue to use the method applied to a sample of HAeBe stars showing PAH emission by Sloan et al. (2005). As they did, we fit line segments to the continuum on either side of each PAH feature and integrate the flux above this estimated continuum to measure the strength of the feature. We determine the wavelength with half the emitted flux to either side, which, for the remainder of this paper, we call the central wavelength.

Other authors have analyzed the position of the peak wavelength, but this result differs from the central wavelength for two reasons. First, PAH features are usually asymmetric, so the peak will lie slightly to the blue of the center. Second, most of the features are blends of multiple transitions. The feature peaking at $\sim 7.7-7.9 \mu \mathrm{m}$ can be analyzed as a combination of features at 7.65 and $7.85 \mu \mathrm{m}$ (e.g., Peeters et al. 2002; Bregman \& Temi 2005). As the $7.85 \mu \mathrm{m}$ component increases with respect to the $7.65 \mu \mathrm{m}$ component, the central wavelength will move steadily to the red, but the peak wavelength will not move significantly until the red component outshines the blue component. Analyz-

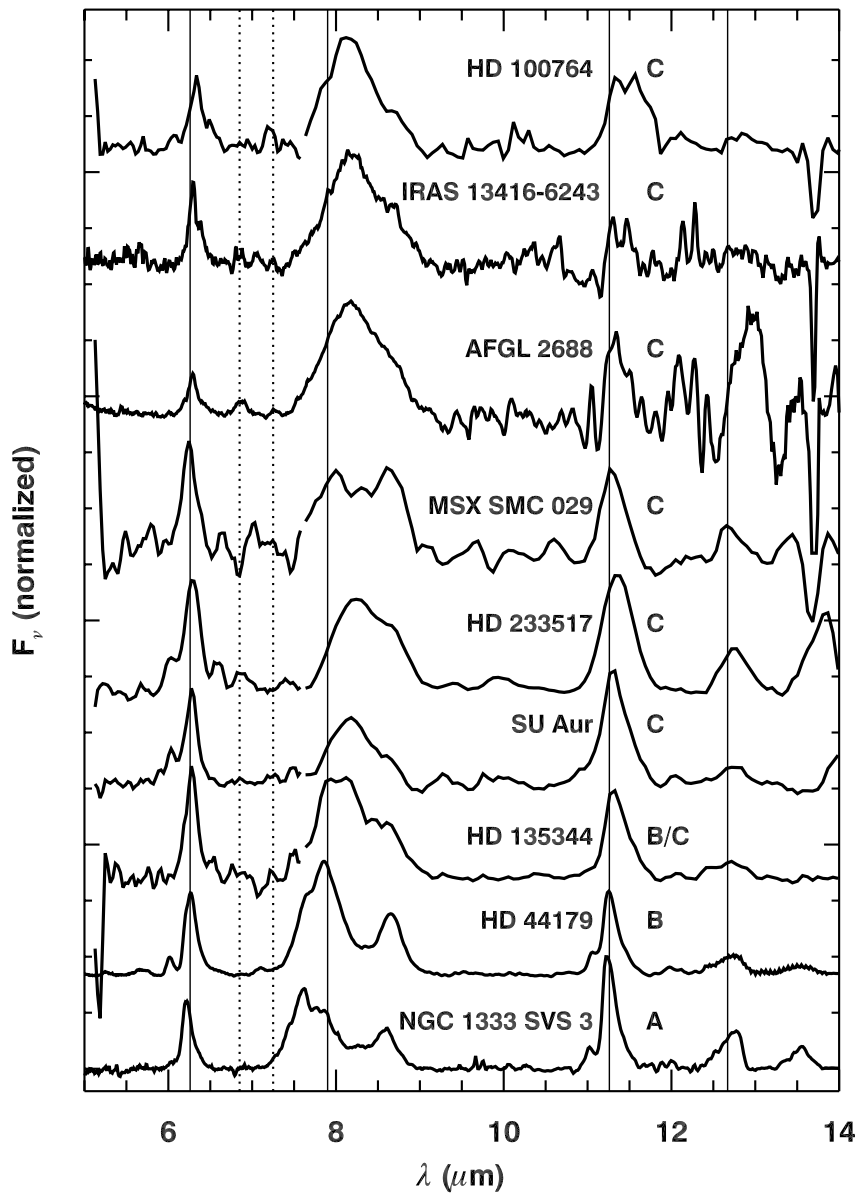

FIG. 4.-Continuum-subtracted spectrum of HD 100764, along with several comparison spectra (also continuum-subtracted). The name of each source is followed by the classification of its PAH spectrum. The vertical lines are at the centers of the PAH features for a class B spectrum: 6.26, 7.90, 11.26, and 12.67 $\mu \mathrm{m}$. The majority of the class $\mathrm{C}$ spectra show features shifted to the red of these lines. The dashed lines at 6.85 and 7.25 mark the positions of aliphatic emission features. The bottom two spectra are the prototypical class A and class B spectra from the ISO/SWS database. The remainder are the sample of known class C PAH spectra (or in the case of HD 135344, nearly class C PAH spectrum). The extractions of the PAH features at 12.0 and $12.7 \mu \mathrm{m}$ in AFGL 2688 are particularly untrustworthy due to their extremely low contrast and resultant sensitivity to the location of the spline points for the continuum. In the spectrum of AFGL 2688, the bottom of the $13.7 \mu \mathrm{m}$ feature has been truncated to prevent it from overrunning the spectra below. [See the electronic edition of the Journal for a color version of this figure.]

ing the central wavelength provides better insight about the relative strengths of the various components of each PAH feature.

Table 3 gives the wavelength ranges used to fit the line segments to each feature; these have changed some from the ranges used by Sloan et al. (2005). We use the class $\mathrm{C}$ wavelengths for all of the sources except HD 44179 and NGC 1333 SVS 3, which require shifted wavelengths to extract the features. All of the spectra except HD 100764 and SU Aur have a feature at $8.6 \mu \mathrm{m}$ from the $\mathrm{C}-\mathrm{H}$ in-plane bending mode on the red shoulder of the $\mathrm{C}-\mathrm{C}$ feature peaking between 7.7 and $8.2 \mu \mathrm{m}$. To eliminate the influence of the $8.6 \mu \mathrm{m}$ feature on the position and strength of the broader feature, we fit a line segment underneath it and include only the flux below that line in our measurement of the 7.7-8.2 $\mu \mathrm{m}$ feature.

Table 4 presents the extracted central wavelengths, while Table 5 gives the equivalent fluxes (i.e., integrated flux densities), and for the acetylene band at $13.7 \mu \mathrm{m}$, the equivalent width. In cases where no feature is apparent or the strength of the extracted 
TABLE 3

Wavelength Intervals Used to Extract Spectral Features

\begin{tabular}{|c|c|c|c|c|}
\hline \multirow[b]{2}{*}{$\begin{array}{l}\text { FeatuRe } \\
(\mu \mathrm{m})\end{array}$} & \multicolumn{2}{|c|}{ For Class C Sources } & \multicolumn{2}{|c|}{ For Class A AND B Sources } \\
\hline & $\begin{array}{l}\lambda_{\text {blue }} \\
(\mu \mathrm{m})\end{array}$ & $\begin{array}{c}\lambda_{\text {red }} \\
(\mu \mathrm{m})\end{array}$ & $\begin{array}{c}\lambda_{\text {blue }} \\
(\mu \mathrm{m})\end{array}$ & $\begin{array}{c}\lambda_{\text {red }} \\
(\mu \mathrm{m})\end{array}$ \\
\hline$\ldots$ & $5.80-5.95$ & $6.65-6.80$ & As class $\mathrm{C}$ & $6.54-6.69$ \\
\hline 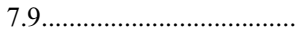 & $7.30-7.60$ & $9.00-9.30$ & $7.10-7.40$ & $8.92-9.17$ \\
\hline 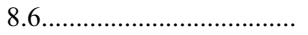 & $8.32-8.44$ & $8.86-8.98$ & As class $\mathrm{C}$ & As class $\mathrm{C}$ \\
\hline$\ldots$ & $10.80-11.00$ & $11.85-11.95$ & $10.68-10.86$ & $11.72-11.90$ \\
\hline 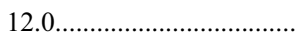 & $11.85-11.95$ & $12.35-12.60$ & $11.72-11.90$ & $12.13-12.25$ \\
\hline 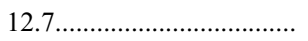 & $12.35-12.60$ & $13.25-13.40$ & $12.07-12.19$ & $12.98-13.10$ \\
\hline 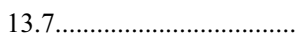 & $13.25-13.55$ & $13.83-14.13$ & As class $\mathrm{C}$ & As class $\mathrm{C}$ \\
\hline
\end{tabular}

feature has a $\mathrm{S} / \mathrm{N}$ ratio less than 1 , Table 4 does not quote a central wavelength. Table 4 includes notes indicating those wavelengths for features with $\mathrm{S} / \mathrm{N}$ ratios less than 2 or 3 . Results for some of the comparison spectra have been published recently, but the results presented here represent newer S14 pipeline output and reflect modifications to the continuum fitting and extraction wavelengths.

\subsection{The PAH Spectra}

Figures 3 and 4 include the spectra of our class A and B prototypes, NGC 1333 SVS 3 and HD 44179. Using these and other SWS data, we find that the $\mathrm{C}-\mathrm{C}$ modes produce features in class A spectra with typical central wavelengths of 6.23 and $7.7 \mu \mathrm{m}$, while class B spectra have features centered at 6.26 and $7.9 \mu \mathrm{m}$ (in essential agreement with Peeters et al. 2002). In the two class $\mathrm{C}$ spectra in the SWS database, these features are shifted to 6.30 and $8.2 \mu \mathrm{m}$, respectively. In HD 100764, they are centered at 6.33 and $8.15 \mu \mathrm{m}$. In the other class C IRS sources, these features appear in the 6.25-6.28 $\mu \mathrm{m}$ and 8.09-8.29 $\mu \mathrm{m}$ ranges.

The features beyond $10 \mu \mathrm{m}$ arise from out-of-plane $\mathrm{C}-\mathrm{H}$ bending modes. The wavelength of these modes depends on the number of adjacent hydrogen atoms on a single aromatic ring (e.g., Allamandola et al. 1989). Single H atoms produce the solo mode, which class A and B spectra show between 11.21 and $11.30 \mu \mathrm{m}$, with a mean at $11.26 \mu \mathrm{m}$. Rings with two adjacent $\mathrm{H}$ atoms produce the duo mode, which normally appears at $12.00 \mu \mathrm{m}$. Three adjacent $\mathrm{H}$ atoms produce the trio mode, which appears at $12.70 \mu \mathrm{m}$ in the class A and B spectra.

In all of the class $\mathrm{C}$ sources, the solo out-of-plane bending mode has shifted to longer wavelengths. In the comparison class C PAH spectra observed by the IRS, the features have shifted from the nominal $11.26 \mu \mathrm{m}$ position to between 11.32 and $11.36 \mu \mathrm{m}$.
In the two class $\mathrm{C}$ spectra in the SWS database, the shifts are difficult to measure, due to possible effects from molecular absorption and artifacts. Our best estimate places the band centers in the $11.36-11.42 \mu \mathrm{m}$ range. In HD 100764, this feature is centered further to the red, at $11.47 \mu \mathrm{m}$.

The duo and trio modes also appear to shift in the class $\mathrm{C}$ spectra, but they are fainter and more difficult to measure. A shift is apparent in both features in the SWS spectra of AFGL 2688 and IRAS 13416, from 12.0 to $\sim 12.1-12.2 \mu \mathrm{m}$ for the duo mode and from 12.7 to $\sim 12.9 \mu \mathrm{m}$ for the trio mode, but these results should be treated with reservation. The problem is that the contrast of these features is small, and their apparent strength and position depend on the details of how we fit a spline to the continuum. Of the IRS comparison spectra, SU Aur shows the clearest duomode feature, but the $\mathrm{S} / \mathrm{N}$ ratio is only $\sim 2$ and the shift is minimal. The spectrum of HD 100764 shows a more significant shift, but again, the $\mathrm{S} / \mathrm{N}$ ratio is only $\sim 2$. The situation with the trio mode is similar in the IRS spectra. A shift is apparent, but the uncertainties in central wavelength are large.

In summary, the positions of the PAH features in the spectrum of HD 100764 are fully consistent with a class C PAH spectrum. Two of the features, at 6.33 and $11.47 \mu \mathrm{m}$, represent extreme positions for our sample. The spectrum is definitely not class A or B.

\subsection{Other Spectral Features}

In Figures 2 and 4, the spectrum of HD 100764 shows structure between the PAH features at 6.3 and $8.1 \mu \mathrm{m}$ reminiscent of the features observed in emission at 6.85 and $7.25 \mu \mathrm{m}$ in the spectra of the HAeBe stars HD 34282 and HD 169142 by Sloan et al. (2005). These features are most obvious in the spectrum of AFGL 2688 (where they can be seen in both scan directions).

TABLE 4

Central Wavelengths of the PAH and $\mathrm{C}_{2} \mathrm{H}_{2}$ Features

\begin{tabular}{|c|c|c|c|c|c|c|c|}
\hline TARGET & \multicolumn{7}{|c|}{$\lambda_{c}(\mu \mathrm{m})$} \\
\hline 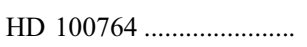 & $6.33 \pm 0.01$ & $8.15 \pm 0.06$ & & $11.47 \pm 0.07$ & $12.14 \pm 0.15^{\mathrm{a}}$ & $12.83 \pm 0.27^{\mathrm{a}}$ & $13.70 \pm 0.02$ \\
\hline 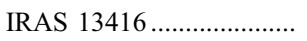 & $6.31 \pm 0.01$ & $8.22 \pm 0.02$ & $8.71 \pm 0.02$ & $11.42 \pm 0.03$ & $12.25 \pm 0.07$ & $12.87 \pm 0.10$ & $13.70 \pm 0.00$ \\
\hline 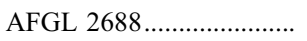 & $6.29 \pm 0.01$ & $8.20 \pm 0.01$ & $8.68 \pm 0.03$ & $11.36 \pm 0.03$ & $12.13 \pm 0.03$ & $12.93 \pm 0.03$ & $13.71 \pm 0.01$ \\
\hline MSX SMC 029................... & $6.25 \pm 0.04$ & $8.12 \pm 0.10$ & $8.68 \pm 0.05$ & $11.32 \pm 0.07$ & $\ldots$ & $\ldots$ & $13.67 \pm 0.02$ \\
\hline 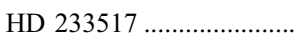 & $6.28 \pm 0.03$ & $8.26 \pm 0.03$ & $8.72 \pm 0.07$ & $11.35 \pm 0.02$ & $\ldots$ & $12.75 \pm 0.09$ & $\ldots$ \\
\hline HD 44179 & $6.26 \pm 0.00$ & $7.84 \pm 0.06$ & $8.66 \pm 0.01$ & $11.27 \pm 0.00$ & $12.00 \pm 0.04$ & $12.71 \pm 0.02$ & $\ldots$ \\
\hline NGC 1333 SVS $3 \ldots \ldots \ldots \ldots$. & $6.23 \pm 0.00$ & $7.68 \pm 0.01$ & $8.61 \pm 0.01$ & $11.26 \pm 0.01$ & $12.00 \pm 0.02$ & $12.70 \pm 0.01$ & $\ldots$ \\
\hline
\end{tabular}

\footnotetext{
${ }^{\text {a }} \mathrm{S} / \mathrm{N}$ of extracted feature $<2$.

b $\mathrm{S} / \mathrm{N}$ of extracted feature $<3$.
} 
TABLE 5

Extracted Strengths of the PAH and $\mathrm{C}_{2} \mathrm{H}_{2}$ Features

\begin{tabular}{|c|c|c|c|c|c|c|c|}
\hline \multirow[b]{2}{*}{ TARGET } & \multicolumn{6}{|c|}{$F\left(10^{-15} \mathrm{~W} \mathrm{~m}^{2}\right)$} & \multirow{2}{*}{$\frac{W_{\lambda}\left(10^{-3} \mu \mathrm{m}\right)^{\mathrm{a}}}{13.7 \mu \mathrm{m}}$} \\
\hline & $6.2 \mu \mathrm{m}$ & $7.7-8.2 \mu \mathrm{m}$ & $8.6 \mu \mathrm{m}$ & $11.3 \mu \mathrm{m}$ & $12.0 \mu \mathrm{m}$ & $12.7 \mu \mathrm{m}$ & \\
\hline HD $100764 \ldots .$. & $4.61 \pm 0.24$ & $9.15 \pm 0.82$ & $\ldots$ & $2.27 \pm 0.28$ & $0.32 \pm 0.17$ & $0.16 \pm 0.13$ & $3.18 \pm 0.64$ \\
\hline 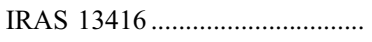 & $57.3 \pm 2.4$ & $225 \pm 5$ & $1.73 \pm 0.51$ & $22.9 \pm 1.5$ & $7.39 \pm 1.66$ & $7.71 \pm 1.54$ & $5.10 \pm 0.48$ \\
\hline 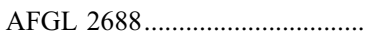 & $111 \pm 4$ & $860 \pm 10$ & $18.6 \pm 1.6$ & $147 \pm 15$ & $76.5 \pm 7.4$ & $204 \pm 14$ & $6.23 \pm 0.57$ \\
\hline 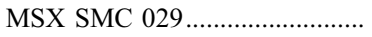 & $0.16 \pm 0.03$ & $0.29 \pm 0.03$ & $0.051 \pm 0.007$ & $0.068 \pm 0.012$ & $0.005 \pm 0.021$ & $0.001 \pm 0.015$ & $14.84 \pm 2.96$ \\
\hline 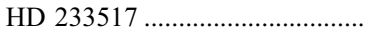 & $1.46 \pm 0.15$ & $1.75 \pm 0.08$ & $0.11 \pm 0.03$ & $0.66 \pm 0.04$ & $\ldots$ & $0.091 \pm 0.037$ & $1.22 \pm 3.09$ \\
\hline 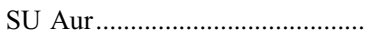 & $6.08 \pm 0.35$ & $6.64 \pm 0.35$ & $0.14 \pm 0.12$ & $3.77 \pm 0.13$ & $0.080 \pm 0.037$ & $0.31 \pm 0.08$ & . \\
\hline 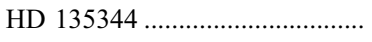 & $5.44 \pm 0.54$ & $9.42 \pm 0.46$ & $0.43 \pm 0.12$ & $2.34 \pm 0.06$ & $\ldots$ & $0.14 \pm 0.04$ & $\ldots$ \\
\hline 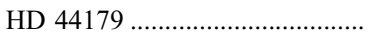 & $2969 \pm 23$ & $5731 \pm 677$ & $960 \pm 19$ & $1182 \pm 19$ & $48.3 \pm 10.6$ & $239 \pm 14$ & $\ldots$ \\
\hline NGC 1333 SVS 3 ........................ & $241 \pm 3$ & $280 \pm 7$ & $69.9 \pm 1.8$ & $172 \pm 5$ & $3.52 \pm 0.63$ & $57.2 \pm 1.2$ & $\ldots$ \\
\hline
\end{tabular}

${ }^{\text {a }}$ Equivalent width of the acetylene absorption band.

The carriers of the 6.85 and $7.25 \mu \mathrm{m}$ features have been identified as aliphatic hydrocarbons (Chiar et al. 2000) and/or hydrogenated amorphous carbon (HAC; Furton et al. 1999). In HD 100764, the features are faint and close to our uncertainty level. The $7.25 \mu \mathrm{m}$ feature is stronger, which is a little unusual. As a consequence, our identification of these features in HD 100764 is only tentative. Other class $\mathrm{C}$ spectra also show possible spectral structure in this area, but with some variations. For example, IRAS 13416 shows an extra feature (all three appear in both scan directions). The class A and B prototypes do not show the 6.85 and $7.25 \mu \mathrm{m}$ features.

Excluding the emission features from PAHs and related molecules, the remaining spectrum from HD 100764 consists of a cool dust continuum, absorption due to acetylene at $13.7 \mu \mathrm{m}$ and a broad emission feature centered at $\sim 10.6 \mu \mathrm{m}$. The acetylene band is centered at $13.7 \mu \mathrm{m}$, which is typical for a carbon star in the Galactic sample, and it has an equivalent width of only $0.0032 \pm 0.0006 \mu \mathrm{m}$, about a factor of 10-20 times weaker than seen in the SWS sample of Galactic carbon stars (Sloan et al. 2006). The acetylene bands are apparent in sources with optically thick dust shells, indicating that the absorbing molecules are not in the stellar photosphere, but instead intermixed with or above the emitting dust (Sloan et al. 2006 and references within). In the case of HD 100764, it is similarly likely that the acetylene absorption is not from the stellar photosphere. Instead, the acetylene is probably distributed in and/or above the disk.

The broad emission feature to the blue of the $11.47 \mu \mathrm{m}$ PAH feature can be fit reasonably well with a Gaussian centered at $10.63 \mu \mathrm{m}$ with a full width at half maximum of $1.37 \mu \mathrm{m}$. As Speck et al. (2005) have explained, typical SiC dust grains emit at $\sim 11.3 \mu \mathrm{m}$, but larger SiC grains emit predominantly at $10.8 \mu \mathrm{m}$, because the contribution from the longitudinal optic at $10.8 \mu \mathrm{m}$ increases with respect to the transverse optic at $11.5 \mu \mathrm{m}$ as the grain size grows. Sloan et al. (2006) may have observed this effect in two carbon stars in the SMC, but they were trying to explain a shift from 11.3 to $11.0 \mu \mathrm{m}$. A shift past $10.8 \mu \mathrm{m}$ to $10.6 \mu \mathrm{m}$ seems far less likely. We conclude that this broad emission feature at $10.6 \mu \mathrm{m}$ is not likely to arise from $\mathrm{SiC}$; it remains unidentified.

\section{DISCUSSION}

\subsection{Dependence of the PAH Emission on Excitation Temperature}

Table 2 gives the spectral types and effective temperatures of our PAH sample. HD 100764 has been classified as R2 or C1,1 (Sanford 1944; Yamashita 1972) and has an effective temperature of $4850 \mathrm{~K}$ (Dominy 1984). The two class C sources in the
SWS database appear to be post-AGB objects evolving into planetary nebulae (PNe). The central star in AFGL 2688 is classified as F5 Ia, and the surrounding nebulosity shows no strong emission lines (Crampton et al. 1975). IRAS 13416-6243 has a spectral type of G1 I (Hu et al. 1993) and also shows no ionized emission (Käufl et al. 1993). ${ }^{8}$ Van der Veen et al. (1989) describe it as in transition from the AGB to a PN. We have determined temperatures from the spectral classes for these two objects using the relation calibrated by McWilliam (1991).

The PAH spectrum of MSX SMC 029 is best described as class C. While the $6.2 \mu \mathrm{m}$ feature has not shifted much from the position seen in class B, the 7-9 $\mu \mathrm{m}$ PAH complex and $11.3 \mu \mathrm{m}$ feature are shifted to the red. Like the post-AGB objects AFGL 2688 and IRAS 13416, MSX SMC 029 shows an absorption band from acetylene at $13.7 \mu \mathrm{m}$. Kraemer et al. (2006) argue that it is one of the few known post-AGB objects in the SMC. It is unfortunate that there are no optical data to help classify the spectrum of the central source.

Sloan et al. (2005) presented the spectra of four HAeBe stars, three classified as early to mid A dwarfs, and one, HD 135344, as F4 Ve (Dunkin et al. 1997), which corresponds to an effective temperature of $6590 \mathrm{~K}$ (using the calibration of Kenyon \& Hartmann 1995, Table A5). The PAHs around the A dwarfs resemble class B spectra, except that the $7.85 \mu \mathrm{m}$ feature is shifted to $7.9-8.0 \mu \mathrm{m}$, and in the F dwarf HD 135344, this feature is shifted even further, to $8.08 \mu \mathrm{m}$, making it intermediate between class B and C. Keller et al. (2007) have re-examined these four spectra as part of a larger sample of $18 \mathrm{HAeBe}$ stars and four related stars, and they confirm these previous results. As a group, the HAeBe stars exhibit PAH spectra which differ from the typical class B PAH spectra, with more redshifted features. The coolest stars in the sample have spectra which are even closer to class C. One source examined by Keller et al. (2007) is the intermediate-mass T Tauri star SU Aur, which has a spectral class of G1 and an effective temperature of $5945 \mathrm{~K}$ (Calvet et al. 2004). Furlan et al. (2006) noted that while silicate emission dominates its infrared spectrum, emission from PAHs is apparent at $6.2 \mu \mathrm{m}$ and on the wings of the $10 \mu \mathrm{m}$ silicate feature at 8 and $11.3 \mu \mathrm{m}$. This source is the coolest star in the sample of Keller et al. (2007), and it is their one source with an unambiguous class $\mathrm{C} \mathrm{PAH}$ spectrum.

HD 233517 has a spectral type of K2 III (Fekel et al. 1996), an effective temperature of $4475 \mathrm{~K}$ (Balachandran et al. 2000), and in the infrared, a class C PAH spectrum. It is the coolest object in

\footnotetext{
${ }^{8}$ Van de Steene et al. (2000) actually detected Br $\gamma$ absorption at $2.17 \mu \mathrm{m}$.
} 


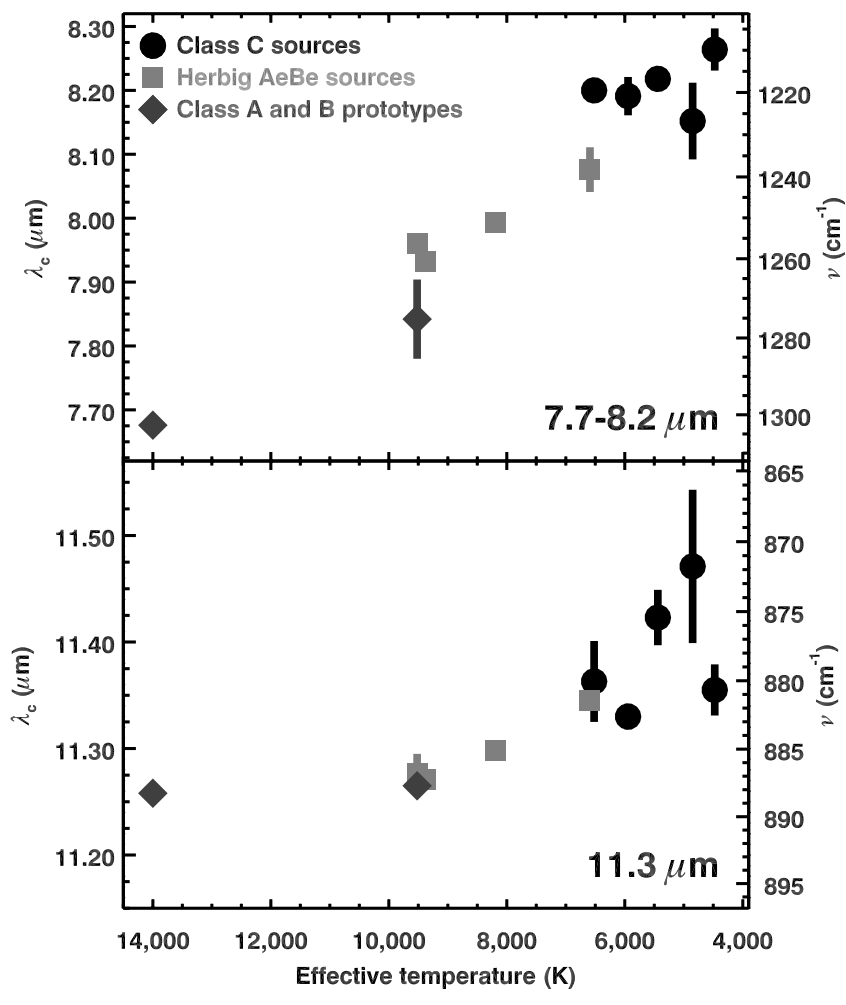

FIG. 5.-Correlation between the central wavelength (defined in $\S 2.3$ ) of the two strongest PAH features and the effective temperature of the exciting star. The top panel plots the position of the 7.7-8.2 $\mu \mathrm{m}$ PAH complex, and the bottom panel plots the position of the $11.3 \mu \mathrm{m}$ feature. This figure contains three Herbig $\mathrm{Ae} / \mathrm{Be}$ stars not included in Tables 2, 4, or 5 . The IRS data for these sources, HD 34282, HD 141569, and HD 169142, appear elsewhere (Sloan et al. 2005; Keller et al. 2007). HD 135344 is the right-most square. The two prototypes are NGC 1333 SVS 3 ( far left) and HD 44179 (second to left). [See the electronic edition of the Journal for a color version of this figure.]

our comparison sample, and the center of its 7-9 $\mu \mathrm{m}$ PAH complex is also at the longest wavelength observed.

By comparison, the class A and B prototypes are warmer. NGC 1333 SVS 3 has a spectral class of B6 (Harvey et al. 1984), which corresponds to an effective temperature of $14,000 \mathrm{~K}$ (Kenyon \& Hartmann 1995). HD 44179 has a spectral type of A0 III (Cohen et al. 1975), corresponding to $9520 \mathrm{~K}$ (Kenyon \& Hartmann 1995). While a range of temperatures have been used to model the Red Rectangle, we adopt $9520 \mathrm{~K}$ as most consistent with the optical spectral class.

Figure 5 plots the positions of the PAH features as a function of the effective temperature of the exciting source. In addition to the comparison sample in Tables 2, 4, and 5, the figure also includes the HAeBe stars HD 34282, HD 141569, and HD 169142. Sloan et al. (2005) first published the IRS spectra of these sources, and the results presented here are from the more recent analysis by Keller et al. (2007). The correlation of the position of the 7.7-8.2 $\mu \mathrm{m}$ PAH complex with effective temperature is impressive. While the $11.3 \mu \mathrm{m}$ PAH feature also tends to shift to longer wavelength as the temperature decreases, the trend is not so clear, with little difference between the class A and B sources and more scatter in the class $\mathrm{C}$ sources.

\subsection{Spatial Dependence of the PAHs in Extended Sources}

In the original PAH model, ultraviolet radiation was required to excite the UIR emission features (e.g., Allamandola et al. 1989). Uchida et al. (1998, 2000) first observed PAH emission in cooler radiation fields, with effective temperatures as low as
$6800 \mathrm{~K}$ in the case of the reflection nebula vdB 133. While these observations initially cast some doubt on the PAH model, Li \& Draine (2002) showed that the absorption of visible photons by large or ionized PAHs can produce the observed UIR features.

Since PAH emission often arises in a photodissociation region (PDR), where a front is eating its way into a denser region and destroying the PAHs in the process, one might expect a change in the nature of the PAH spectrum as the temperature of the exciting radiation field varies. Uchida et al. (2000) did not observe variation in the relative strengths of the features in their sample, but they did observe a change in the shape of the 7-9 $\mu \mathrm{m}$ PAH complex.

Bregman \& Temi (2005) analyzed the shape of the 7-9 $\mu \mathrm{m}$ complex as a function of position within the reflection nebulae vdB 133, NGC 1333 SVS 3, and vdB 17 (also within NGC 1333). They found that closer to the exciting star, the strength of the $7.65 \mu \mathrm{m}$ component increases relative to the $7.85 \mu \mathrm{m}$ component, thus shifting the measured center of the 7.7-7.9 $\mu \mathrm{m} \mathrm{PAH}$ feature to the blue. They also found that this complex grows stronger relative to the $\mathrm{C}-\mathrm{H}$ modes at longer wavelengths near the exciting sources and suggested that the wavelength shift at 7.7-7.9 $\mu \mathrm{m}$ might be related either to the ionization fraction of the PAHs or to PAH processing by the radiation field, as previously proposed by Hony et al. (2001).

\subsection{The Possible Role of Aliphatics}

Hydrocarbons can exist in both aromatic or aliphatic forms. Aromatic hydrocarbons are dominated by $s p^{2}$ bonds which produce the polycyclic ring structure that define PAHs. Aliphatics include $s p^{1}$ bonds, as seen in acetylene, and $s p^{3}$ bonds, as seen in methane and longer alkane chains. The $s p^{2}$ bonds efficiently spread the energy absorbed from a photon over the entire molecule before it can disrupt a single bond, making the aromatic carbon structure in PAHs more stable than aliphatic bonding structures. Thus, when a mixture of aliphatic and amorphous hydrocarbons is exposed to a harsh radiation environment, we expect the aliphatic bonds to be broken first. We propose that the class $\mathrm{C}$ spectra arise from mixtures of aromatic and aliphatic material that have not yet been subjected to intense ultraviolet radiation, allowing the more fragile aliphatic bonds to survive.

Consider the class $\mathrm{C}$ spectrum of MSX SMC 029. This source also shows a strong absorption band from acetylene $\left(\mathrm{C}_{2} \mathrm{H}_{2}\right)$ at $13.7 \mu \mathrm{m}$, centered within a broader absorption band in the $12-$ $16 \mu \mathrm{m}$ region (see Kraemer et al. 2006, Fig. 4). Kraemer et al. (2006) argue that this broader band is due to other aliphatic molecules seen in a similar spectrum at higher spectral resolution in SMP LMC 11, a source in the Large Magellanic Cloud in transition to the early planetary nebula stage (Bernard-Salas et al. 2006, Fig. 1). Acetylene appears in other class C PAH spectra, including AFGL 2688, IRAS 13146, and our spectrum of HD 100764. ${ }^{9}$ The association of the acetylene absorption band at $13.7 \mu \mathrm{m}$ and the broader $12-16 \mu \mathrm{m}$ absorption with class $\mathrm{C} \mathrm{PAH}$ emission is consistent with our proposal that the class $\mathrm{C}$ spectra arise from mixtures of PAHs and aliphatics.

The ground-based mid-infrared spectrum of HD 56126 (IRAS 07134+1005) shows redshifted PAH features similar to our IRS spectrum of HD 100764, with emission both in a plateau at $8.0-8.2 \mu \mathrm{m}$ and from out-of-plane bending modes at 11.4 and $12.2 \mu \mathrm{m}$ (Justtanont et al. 1996). Although not as pronounced, the infrared spectra of IRAS $04296+3429$ and IRAS 05341+ 0852 display similar unusual characteristics. All three of these sources are post-AGB objects. HD 56126 is classified as F5 I

\footnotetext{
9 It is missing from HD 233517, which is an oxygen-rich source, and the disks around HD 135344 and SU Aur.
} 
(Nassau et al. 1965), while IRAS 05341 and IRAS 04296 are F4 Iab: and G0 Ia, respectively. These sources also show unusual spectra in the $3 \mu \mathrm{m}$ range, which is dominated by $\mathrm{C}-\mathrm{H}$ stretching modes. IRAS 05341 shows a feature at $3.40 \mu \mathrm{m}$ that dominates the normally stronger feature at $3.29 \mu \mathrm{m}$ (Geballe \& van der Veen 1990), while IRAS 04296 also shows an unusually strong $3.40 \mu \mathrm{m}$ component (Geballe et al. 1992). The $3.29 \mu \mathrm{m}$ feature arises from an aromatic $\mathrm{C}-\mathrm{H}$ stretching mode (e.g., Duley \& Williams 1981; Allamandola et al. 1989), while the $3.4 \mu \mathrm{m}$ feature arises from a similar mode in aliphatic $\mathrm{C}-\mathrm{H}$ bonds (Duley \& Williams 1981; Jourdain de Muizon et al. 1990; Geballe et al. 1994).

Geballe et al. (1989) observed an increase in the ratio of the aliphatic $3.4 \mu \mathrm{m}$ feature to the aromatic $3.29 \mu \mathrm{m}$ feature in the shielded molecular zone of the Orion Bar compared with the ionized zone. Sloan et al. (1997) verified these observations with long-slit $3 \mu \mathrm{m}$ spectroscopy and showed that the $3.4 \mu \mathrm{m}$ feature actually arises from multiple aliphatic components. Joblin et al. (1996) obtained spatially resolved $3 \mu \mathrm{m}$ spectroscopy in the reflection nebulae NGC 1333 and NGC 2023 and found that where the ultraviolet radiation field was less intense, the $3.4 \mu \mathrm{m}$ feature was stronger compared to the $3.29 \mu \mathrm{m}$ feature. All of these authors argued that the radiation field is altering an initial mix of aliphatic and aromatic bonds by destroying the more fragile aliphatic bonds.

\subsection{Hydrogenated Amorphous Carbon}

Hydrogenated amorphous carbon (HAC) is a generic name for a mixture of aliphatic and aromatic carbon, consisting of PAH clusters embedded within a matrix of aliphatically bonded material (see Jones et al. 1990 and references therein). This description is similar to the material we are proposing to explain the class C PAH spectra.

Compared to previously obtained class C PAH spectra, HD 100764 represents an extreme case, because its features are more consistently redshifted and its exciting radiation field is one of the coolest known. The PAHs around this source must be relatively unprocessed by energetic photons, making this source an excellent candidate for the presence of HAC-like structures.

Figure 6 shows two representative laboratory spectra of a HAC sample at $\sim 800 \mathrm{~K}$ (Scott 1997). HAC films with a thickness of $\sim 1 \mu \mathrm{m}$ were prepared by ablating graphite in a hydrogen atmosphere with an excimer laser, as reported previously (Scott \& Duley 1996; Scott et al. 1997a). Films were deposited on a stainless steel substrate, placed in a vacuum chamber, and heated by conduction to $\sim 800 \mathrm{~K}$. Infrared emission spectra were obtained with a Fourier-transform spectrometer at a resolution of $4 \mathrm{~cm}^{-1}$ over a spectral range of $500-6000 \mathrm{~cm}^{-1}(1.7-20 \mu \mathrm{m})$. The spectra were calibrated by taking the ratio of the emission from HAC to that of an uncoated stainless steel substrate at the same temperatures and making a cubic polynomial baseline correction. The two spectral segments from 5.5 to $8.0 \mu \mathrm{m}$ and 7.5 to $14 \mu \mathrm{m}$ in Figure 6 were obtained separately, and their relative intensities are unknown. We have simply scaled both segments to a maximum of 1.0 arbitrarily in the figure.

While the relative band strengths in Figure 6 are not well determined, the positions are more clear. The emission features have shifted to the red, with the nominal 6.23 and $11.26 \mu \mathrm{m}$ features appearing at 6.32 and $11.45 \mu \mathrm{m}$, respectively. If the features in the $12-13 \mu \mathrm{m}$ range can be attributed to the duo and trio $\mathrm{C}-\mathrm{H}$ out-of-plane bending modes, then they have shifted from 11.99 and $12.67 \mu \mathrm{m}$ to 12.39 and $13.43 \mu \mathrm{m}$. The feature centered at $8.53 \mu \mathrm{m}$ could be a redshifted $\mathrm{C}-\mathrm{C}$ mode, or it could simply be the $\mathrm{C}-\mathrm{H}$ in-plane bending mode. This laboratory

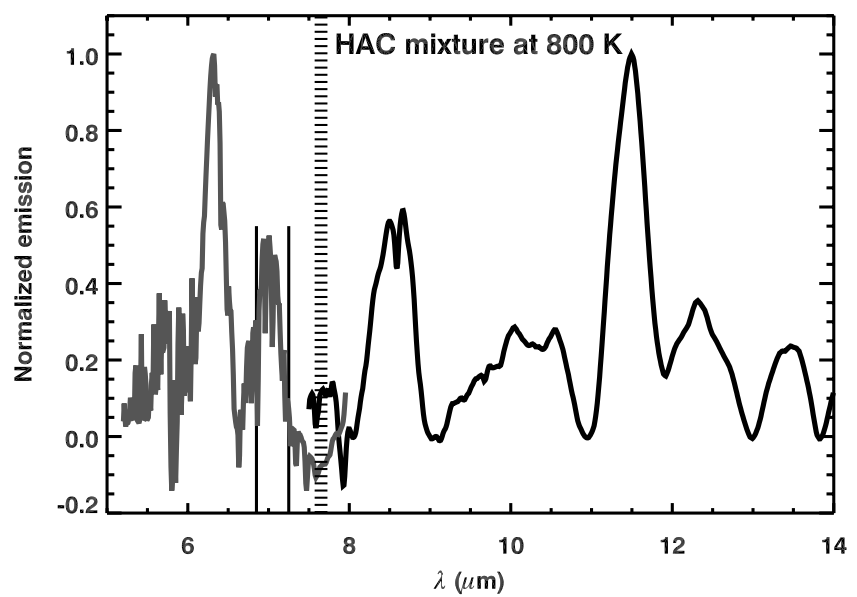

FIG. 6.- Laboratory spectrum of hydrogenated amorphous carbon heated to $800 \mathrm{~K}$ (Scott 1997). The two portions of the spectrum separated by the vertical hatched line at $7.5 \mu \mathrm{m}$ were obtained separately and have been arbitrarily normalized here to the same vertical range. The apparent relative strengths of the two halves are not meaningful. The positions of the bands, however, are a good indication of how the wavelengths of the various modes responsible for the observed PAH features may shift as aliphatic bonds are attached to the aromatic hydrocarbons that form the basis of free PAH molecules. Specifically, the two strongest features at 6.32 and $11.45 \mu \mathrm{m}$ have shifted from 6.23 and $11.26 \mu \mathrm{m}$ in more normal PAH spectra. The vertical lines mark the positions of the 6.85 and $7.25 \mu \mathrm{m}$ features seen in spectra of cooler HAC samples. [See the electronic edition of the Journal for a color version of this figure.]

HAC spectrum is consistent with the wavelength shifts we have observed in class $\mathrm{C}$ PAH sources.

\subsection{PAHs, HAC, and the Class C Spectrum}

Bonds in aromatic and aliphatic species have resonances at different wavelengths (see Bellamy 1978 and the many references therein). In the case of the $\mathrm{C}-\mathrm{H}$ stretching fundamental, the shift from aromatic to aliphatic is always to the red, from 3.29 to $3.40 \mu \mathrm{m}$, but for other vibrational modes, the shifts can be in either direction, or both, depending on the molecules in question. We hypothesize that at longer wavelengths, the substitution of aliphatic sidegroups on the edges of large PAH molecules shifts the out-of-plane aromatic bending modes to the red. While laboratory data of large PAHs with aliphatic substitutions are lacking, data for some of the simplest PAHs are available, and as Figure 7 shows, are consistent with our hypothesis.

Using data from the NIST Chemistry WebBook, maintained by the National Institute for Standards and Technology, ${ }^{10}$ we show in Figure 7 the spectra of naphthalene and 1,2-dimethylbenzene, which is benzene with two adjacent substitutions of methyl sidegroups. The two spectra in Figure 7 were obtained from vapor samples using a Fourier-transform infrared spectrometer at a resolution of $4 \mathrm{~cm}^{-1}$ (Coblentz Society, Inc. 2005). The napthalene was at a temperature of $518 \mathrm{~K}$, while the 1,2-dimethylbenzene was at $423 \mathrm{~K}$. Since both molecules have four adjacent $\mathrm{H}$ atoms on each ring, the only out-of-plane $\mathrm{C}-\mathrm{H}$ bending mode they show in the 11-14 $\mu \mathrm{m}$ region is the quartet mode. This mode has shifted from $12.8 \mu \mathrm{m}$ in naphthalene to $13.5 \mu \mathrm{m}$ in the simpler benzene molecule with the methyl sidegroups, consistent with the anticipated effect of the substitution of a methyl sidegroup. However, this spectral difference may also result from the absence of the second ring in naphthalene, making further analysis of larger molecules highly desirable.

10 At http://webbook.nist.gov/chemistry/. 


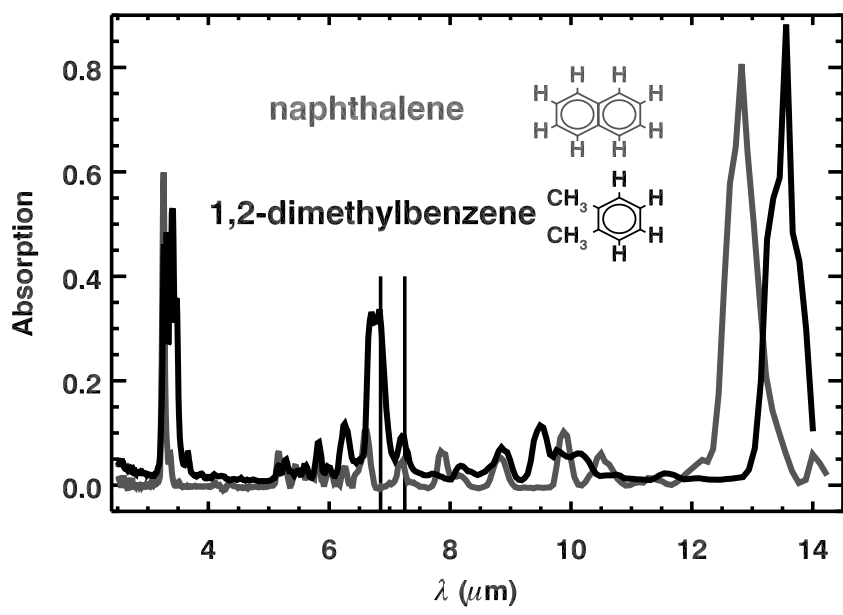

FIG. 7.- Laboratory spectra of two simple PAHs: naphthalene and benzene with two adjacent methyl side groups (from Coblentz Society, Inc. 2005). The substitution of aliphatic sidegroups on benzene adds a second $\mathrm{C}-\mathrm{H}$ stretching mode at $3.4 \mu \mathrm{m}$, produces a strong absorption at $6.8 \mu \mathrm{m}$, shifts the quartet out-ofplane $\mathrm{C}-\mathrm{H}$ bending mode from 12.8 to $13.5 \mu \mathrm{m}$, and produces more spectral structure in the vicinity of the observed 6.85 and $7.25 \mu \mathrm{m}$ aliphatic features (solid vertical lines). [See the electronic edition of the Journal for a color version of this figure.]

We also note that the presence of aliphatic $\mathrm{C}-\mathrm{H}$ stretching modes in the methyl sidegroups on the benzene molecule has added emission at $3.4 \mu \mathrm{m}$ not present in the naphthalene. Furthermore, in the $6-8 \mu \mathrm{m}$ region, the strongest emission feature in naphthalene is at $6.6 \mu \mathrm{m}$, but a stronger feature at $6.8 \mu \mathrm{m}$ is apparent in the methyl-substituted benzene. As described by Bellamy (1978), the carbon skeletal breathing modes in aromatics cluster in the 6.2 to $6.9 \mu \mathrm{m}$ range, while similar modes from methyl $\left(\mathrm{CH}_{3}\right)$ and methylene $\left(\mathrm{CH}_{2}\right)$ groups cluster in the $6.8-$ $7.5 \mu \mathrm{m}$ region. As noted in $\S 2.4$, we may have detected these methyl and methylene features in emission in some of the class C PAH spectra, including HD 100764.

Laboratory HAC spectra by Furton et al. (1999) clearly show emission features at 6.85 and $7.25 \mu \mathrm{m}$. The HAC spectrum in Figure 6 only shows a single emission feature at $7.0 \mu \mathrm{m}$ at about half the strength of the $6.3 \mu \mathrm{m}$ feature. The differences in the spectra probably arise in how the samples were treated and measured. Furton et al. (1999) obtained absorption spectra from samples at $300 \mathrm{~K}$, while the HAC spectrum in Figure 6 was observed in emission at $\sim 800 \mathrm{~K}$. This higher temperature has probably modified the simple groups responsible for the 6.85 and $7.25 \mu \mathrm{m}$ features, and it has also enhanced the $\mathrm{C}-\mathrm{C}$ modes at $6.2 \mu \mathrm{m}$.

HAC samples have a number of degrees of freedom, including the ratio of aliphatic and aromatic bonds and the size distribution and structure of the embedded PAHs, among others, so it is not surprising that no HAC sample matches the observed astrophysical spectra in detail. It is worth recalling that no laboratory $\mathrm{PAH}$ spectrum provides a precise match to astrophysical PAH spectra either. Available data are consistent with the hypothesis that increasing the ratio of aliphatic to aromatic bonds in a hydrocarbon mixture explains the differences between class B and class C PAH spectra.

We propose that complex astrophysical hydrocarbons are originally synthesized as some form of HAC. When this material is exposed to harsh radiation fields, the aliphatic bonds are destroyed and only free PAHs remain. Scott et al. (1997b) provide evidence that this process could occur. They exposed HAC mixtures to UV radiation and found that the dissolution of the $\mathrm{HAC}$ led to the production of large PAH molecules with attached aliphatic sidegroups. Hu et al. (2006) have recently shown that these mixtures of aromatic and aliphatic bonds have the potential for reproducing the observed features in the 6-9 $\mu \mathrm{m}$ range. These modified PAHs have less than a few hundred atoms and still respond to the absorption of single photons like more typical free PAHs do.

Two separate processes are responsible for the PAH spectra we observe. First, the PAHs must be freed from the aliphatic matrix in which they are initially embedded. Second, the features must be excited. This latter step requires less energy than that needed to completely strip the PAHs of all aliphatic side-chains. The positions of the observed PAH features depend on how completely the PAHs have been freed from the underlying matrix. In the class $\mathrm{C}$ PAH sources, the aliphatic side groups have only been partially destroyed.

The sample of isolated HAeBe stars represents a stage in the evolution of PAHs between class C and class B. The $7.85 \mu \mathrm{m}$ feature is shifted measurably to $7.9-8.0 \mu \mathrm{m}$ or beyond (Sloan et al. 2005; Keller et al. 2007). Here again, the PAHs may not be entirely stripped from the underlying aliphatic matrix, since the A and $\mathrm{F}$ stars exciting the PAH spectrum may not produce enough photons with sufficient energy to break all of the aliphatic bonds.

\subsection{The Problem of the Reflection Nebulae}

Reflection nebulae typically do not show class C PAH spectra, even though the exciting source can be as cool as $6800 \mathrm{~K}$ (Uchida et al. 1998). The two reflection nebulae in the sample examined by Peeters et al. (2002) are both class A, as are the three spectra presented by Uchida et al. (2000). The objects studied by Bregman \& Temi (2005) include one from Uchida et al., and all three showed variations in the position of the 7.7-7.9 $\mu \mathrm{m}$ feature with radiation field, but not enough to move them even as far as a true class B spectrum.

We suggest that while the PAHs in these complex, evolving environments are now subject only to cool radiation fields, they may have been exposed in the recent past to a large dose of more intense ultraviolet radiation. Reflection nebulae are typically diffuse and are associated with complicated star-forming environments. This diffuse nature means that the PAHs are poorly shielded from both current and past radiation fields. Furthermore, PAHs in a diffuse environment are not able to regenerate any broken aliphatic bands.

The typical PAH emission source is a PDR in a star formation region, and the emitting materials in these environments must have a complicated history of UV irradiation. This history blurs the link between the current PAH composition and the current radiation field, which is probably why the correlation illustrated in Figure 5 has not been noticed before. Only in more isolated sources, such as those examined here, is the link clear.

\section{CONCLUSIONS}

The PAH spectrum of HD 100764 adds to the small, but growing sample of class C PAH spectra, as defined by Peeters et al. (2002). It has one of the coolest exciting stars and some of the most redshifted features in the class. Since all seven of the known class C spectra are excited by relatively cool stars of spectral class $\mathrm{F}$ or later, we argue that the hydrocarbons in these systems are unusual because they have not been exposed to much ultraviolet radiation.

We hypothesize that carbonaceous materials are synthesized as large HAC conglomerates and that aliphatic bonds are subsequently broken in harsh radiation fields. We suggest that PAH spectra evolve over time; the hydrocarbons in class $\mathrm{C}$ objects are 
relatively protected and unprocessed, while class B and A PAHs have been exposed to more energetic photons and are more processed.

We are grateful to the referee, whose careful reading and thoughtful comments led to considerable improvements in the manuscript. We would also like to thank T. Geballe and P. Roche for useful comments and suggestions and M. Werner for generously providing us with his high-resolution spectra of HD 100764.
These observations were made with the Spitzer Space Telescope, which is operated by JPL, California Institute of Technology, under NASA contract 1407 and supported by NASA through JPL (contract 1257184). Support for this work was provided by NASA through contract 960803 issued by JPL/Caltech. W. M. D. is supported by a grant from the NSERC of Canada. A. L. is supported in part by the NASA/Spitzer theory program. This research has made use of the SIMBAD database and VizieR tool for catalog access, operated at the Centre de Données astronomiques de Strasbourg.
Alksnis, A., Balklavs, A., Dzervitis, U., \& Eglitis, I. 1998, A\&A, 338, 209

Allamandola, L. J., Hudgins, D. M., \& Sandford, S. A. 1999, ApJ, 511, L115

Allamandola, L. J., Tielens, A. G. G. M., \& Barker, J. R. 1985, ApJ, 290, L25 . 1989, ApJS, 71, 733

Balachandran, S. C., Fekel, F. C., Henry, G. W., \& Uitenbroek, H. 2000, ApJ, 542,978

Bellamy, L. J. 1978, The Infrared Spectra of Complex Molecules (London: Chapman \& Hall)

Bernard-Salas, J., Peeters, E., Sloan, G. C., Cami, J., \& Houck, J. R. 2006, ApJ, 652, L29

Bregman, J., \& Temi, P. 2005, ApJ, 621, 831

Calvet, N., Muzerolle, J., Briceño, C., Hernández, J., Hartmann, L., Saucedo, J. L., \& Gordon, K. D. 2004, AJ, 128, 1294

Chiar, J. E., Tielens, A. G. G. M., Whittet, D. C. B., Schutte, W. A., Boogert, A. C. A., Lutz, D., van Dishoeck, E. F., \& Bernstein, M. P. 2000, ApJ, 537, 749

Coblentz Society, Inc. 2005, in NIST Chemistry WebBook, NIST Standard Reference Database Number 69, ed. P. J. Linstrom \& W. G. Mallard (Gaithersburg: Natl. Inst. Stan. and Technol.), http://webbook.nist.gov

Cohen, M., et al. 1975, ApJ, 196, 179

Crampton, D., Cowley, A. P., \& Humphreys, R. M. 1975, ApJ, 198, L135

Dominy, J. F. 1984, ApJS, 55, 27

Duley, W. W., \& Williams, D. A. 1981, MNRAS, 196, 269

Dunkin, S. K., Barlow, M. J., \& Ryan, S. G. 1997, MNRAS, 286, 604

Fekel, F. C., Webb, R. A., White, R. J., \& Zuckerman, B. 1996, ApJ, 462, L95

Furlan, E., et al. 2006, ApJS, 165, 568

Furton, D. G., Laiho, J. W., \& Witt, A. N. 1999, ApJ, 526, 752

Geballe, T. R., Joblin, C., d'Hendecourt, L. B., Jourdain de Muizon, M., Tielens, A. G. G. M., \& Leger, A. 1994, ApJ, 434, L15

Geballe, T. R., Tielens, A. G. G. M., Allamandola, L. J., Moorhouse, A., \& Brand, P. W. J. L. 1989, ApJ, 341, 278

Geballe, T. R., Tielens, A. G. G. M., Kwok, S., \& Hrivnak, B. J. 1992, ApJ, 387, L89

Geballe, T. R., \& van der Veen, W. E. C. J. 1990, A\&A, 235, L9

Gillett, F. C., Forrest, W. J., \& Merrill, K. M. 1973, ApJ, 183, 87

Harvey, P. M., Wilking, B. A., \& Joy, M. 1984, ApJ, 278, 156

Hony, S., Van Kerckhoven, C., Peeters, E., Tielens, A. G. G. M., \& Allamandola, L. J. 2001, A\&A, 370, 1030

Houck, J. R., et al. 2004, ApJS, 154, 18

Hu, A., Alkhesho, I., \& Duley, W. W. 2006, ApJ, 653, L157

Hu, J. Y., Slijkhuis, S., de Jong, T., \& Jiang, B. W. 1993, A\&AS, 100, 413

Joblin, C., Tielens, A. G. G. M., Allamandola, L. J., \& Geballe, T. R. 1996, ApJ, 458, 610

Jones, A. P., Duley, W. W., \& Williams, D. A. 1990, QJRAS, 31, 567

Jourdain de Muizon, M., d'Hendecourt, L. B., \& Geballe, T. R. 1990, A\&A, 235,367
Jura, M. 2003, ApJ, 582, 1032

Jura, M., Bohac, C. J., Forrest, W. J., Green, J., Watson, D. M., Sloan, G. C., Markwick-Kemper, F., \& Najita, J. 2006, ApJ, 637, L45

Justtanont, K., Barlow, M. J., Skinner, C. J., Roche, P. F., Aitken, D. K., \& Smith, C. H. 1996, A\&A, 309, 612

Käufl, H. U., Renzini, A., \& Stanghellini, L. 1993, ApJ, 410, 251

Keller, L. D., et al. 2007, ApJ, submitted

Kenyon, S. J., \& Hartmann, L. 1995, ApJS, 101, 117

Kraemer, K. E., Sloan, G. C., Bernard-Salas, J., Price, S. D., Egan, M. P., \& Wood, P. R. 2006, ApJ, 652, L25

Leger, A., \& Puget, J. L. 1984, A\&A, 137, L5

LeVan, P. D., Sloan, G. C., Little-Marenin, I. R., \& Grasdalen, G. L. 1992, ApJ, 392, 702

Li, A., \& Draine, B. T. 2002, ApJ, 572, 232

Lloyd Evans, T. 1990, MNRAS, 243, 336

McClure, R. D. 1997, PASP, 109, 256

McWilliam, A. 1991, AJ, 101, 1065

Nassau, J. J., Stephenson, C. B., \& MacConnell, D. J. 1965, in Luminous Stars in the Northern Milky Way, Vol. 6 (Bergedorf: Hamburger Sternw. \& Warner Swasey Obs.)

Parthasarathy, M. 1991, A\&A, 247, 429

Peeters, E., Hony, S., Van Kerckhoven, C., Tielens, A. G. G. M., Allamandola, L. J., Hudgins, D. M., \& Bauschlicher, C. W. 2002, A\&A, 390, 1089

Sanford, R. F. 1944, ApJ, 99, 145

Scott, A. 1997, Ph.D. thesis, Univ. Waterloo

Scott, A., \& Duley, W. W. 1996, ApJ, 472, L123

Scott, A., Duley, W. W., \& Jahani, H. R. 1997a, ApJ, 490, L175

Scott, A., Duley, W. W., \& Pinho, G. P. 1997b, ApJ, 489, L193

Skinner, C. J. 1994, MNRAS, 271, 300

Sloan, G. C., Bregman, J. D., Geballe, T. R., Allamandola, L. J., \& Woodward, C. E. 1997, ApJ, 474, 735

Sloan, G. C., Kraemer, K. E., Matsuura, M., Wood, P. R., Price, S. D., \& Egan, M. P. 2006, ApJ, 645, 1118

Sloan, G. C., Kraemer, K. E., Price, S. D., \& Shipman, R. F. 2003, ApJS, 147, 379

Sloan, G. C., et al. 2005, ApJ, 632, 956

Speck, A. K., Thompson, G. D., \& Hofmeister, A. M. 2005, ApJ, 634, 426

Uchida, K. I., Sellgren, K., \& Werner, M. 1998, ApJ, 493, L109

Uchida, K. I., Sellgren, K., Werner, M. W., \& Houdashelt, M. L. 2000, ApJ, 530,817

van der Veen, W. E. C. J., Habing, H. J., \& Geballe, T. R. 1989, A\&A, 226, 108 Van de Steene, G. C., van Hoof, P. A. M., \& Wood, P. R. 2000, A\&A, 362, 984 Werner, M. W., et al. 2004, ApJS, 154, 1

Yamashita, Y. 1972, Ann. Tokyo. Astron. Obs., 13, 169 